\title{
Ribosome as a Translocase and Helicase
}

\author{
Chen Bao $^{1, \mathrm{a}}$ and Dmitri N. Ermolenko ${ }^{1, \mathrm{~b} *}$ \\ ${ }^{1}$ Department of Biochemistry \& Biophysics, School of Medicine and Dentistry and Center for RNA Biology, \\ University of Rochester, Rochester, NY, USA \\ a e-mail: Chen_Bao@urmc.rochester.edu \\ ${ }^{b}$ e-mail:dmitri_ermolenko@urmc.rochester.edu
}

Received May 10, 2021

Revised May 21, 2021

Accepted May 21, 2021

\begin{abstract}
During protein synthesis, ribosome moves along mRNA to decode one codon after the other. Ribosome translocation is induced by a universally conserved protein, elongation factor $G(E F-G)$ in bacteria and elongation factor 2 (EF-2) in eukaryotes. EF-G-induced translocation results in unwinding of the intramolecular secondary structures of mRNA by three base pairs at a time that renders the translating ribosome a processive helicase. Professor Alexander Sergeevich Spirin has made numerous seminal contributions to understanding the molecular mechanism of translocation. Here, we review Spirin's insights into the ribosomal translocation and recent advances in the field that stemmed from Spirin's pioneering work. We also discuss key remaining challenges in studies of translocase and helicase activities of the ribosome.
\end{abstract}

DOI: $10.1134 / \mathrm{S} 0006297921080095$

Keywords: ribosome, translocation, helicase, power stroke, Brownian ratchet

\section{INTRODUCTION}

During the elongation phase of protein synthesis, the ribosome decodes sequences of codons by binding of the tRNA molecules charged with amino acids. Both small ( $30 \mathrm{~S}$ in bacteria) and large (50S in bacteria) subunits contain three tRNA binding sites: the A (aminoacyl), the P (peptidyl) and the E (exit) sites. At the beginning of elongation cycle, the newly arrived aminoacyl-tRNA binds to the A site of the ribosome. Following the peptidyl transfer reaction, the resulting peptidyl- and deacylated tRNAs together with associated mRNA codons are translocated from the A and P to P and E sites, respectively (Fig. 1, a-f). The process is catalyzed by a universally conserved protein factor EF-G (EF-2 in eukaryotes). The molecular mechanism of translocation has fascinated scientists since the inception of the proteins synthesis field and remained one of the main areas of research in the laboratory of Alexander Spirin for over 50 years [1-6]. Spirin's laboratory has discovered a number of important facets of the translocation mechanism [7-11]. Spirin's lockingunlocking [2,6] and Brownian ratchet $[4,12,13]$ models of ribosomal translocation were highly influential and provided framework for investigations of translocation for

Abbreviations: EF-G, elongation factor G.

* To whom correspondence should be addressed. decades. Below we review key contributions of the Alexander Spirin's laboratory and recent progress in investigations of ribosomal translocation that stemmed from the Spirin's ideas. More comprehensive reviews of the current state of understanding of the translocation mechanism can be found elsewhere [14-16].

\section{ROLES OF EF-G AND tRNAs IN RIBOSOME TRANSLOCATION ALONG mRNA}

Relative to the uncatalyzed reaction, the universally conserved GTPase, EF-G, accelerates the rate of ribosome translocation by $\sim$ four orders of magnitude [18-20]. GTP hydrolysis by EF-G is activated by the interaction of the $\mathrm{G}$ domain of this protein with the sarcin-ricin loop (SRL) of the 23S rRNA [21, 22]. Works of Kaziro's and Spirin's laboratories established the role of GTP hydrolysis in translocation [9, 23-25]. They demonstrated that EF-G induces efficient translocation in the GTPbound form. In the presence of GDP or in the absence of nucleotides, EF-G does not promote translocation. Replacing GTP with non-hydrolysable analogues preserves the ability of EF-G to induce translocation that was measured by the increase in puromycin reactivity of peptidyl-tRNA or by the release of deacylated tRNA from the ribosome. However, the non-hydrolysable ana- 
a

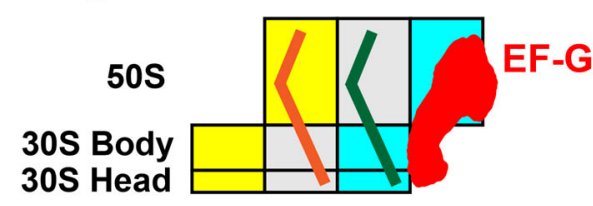

d

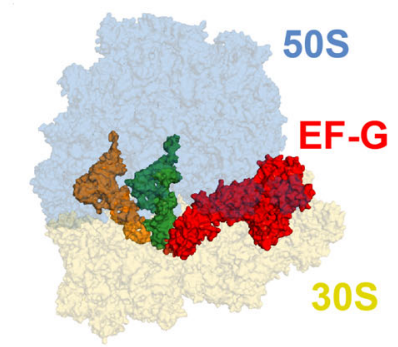

$\mathrm{g}$

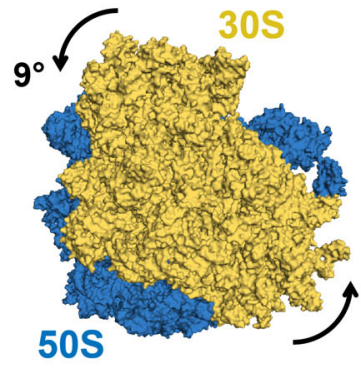

R 70S b

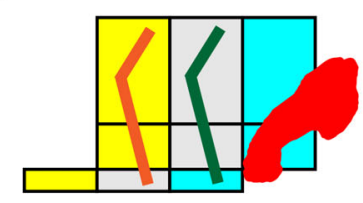

e
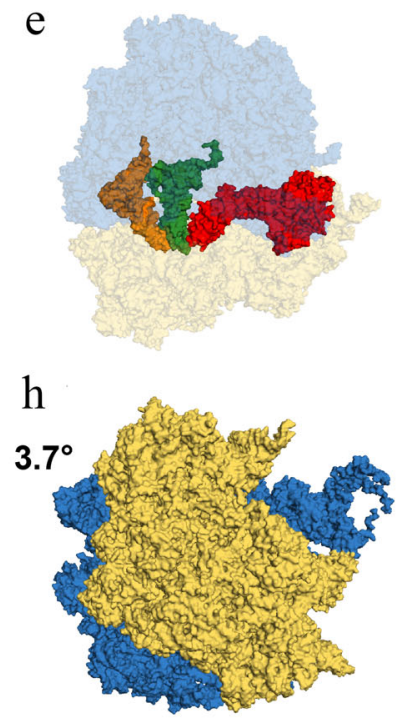

CH 70 S c

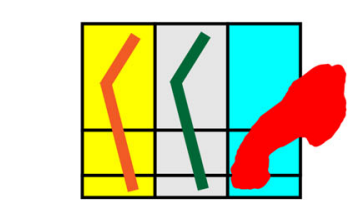

f

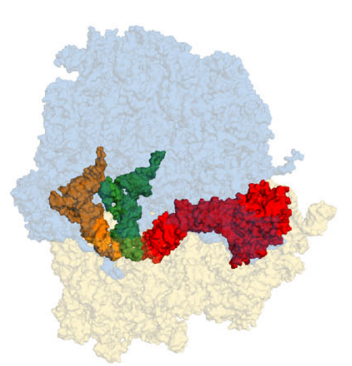

$\mathrm{i}$

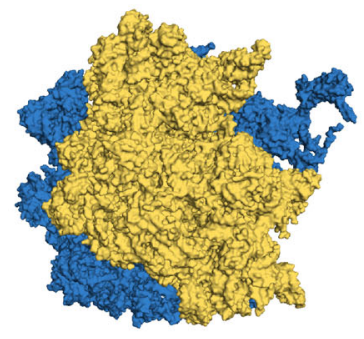

NR 70S

Fig. 1. tRNA movements and conformational rearrangements of the ribosome in EF-G-ribosome complexes. a-c) Box diagrams showing positions of peptidyl- (green) and deacylated (orange) tRNAs relative to the A (cyan), P (grey), and E (yellow) sites on the 50S subunit and $30 \mathrm{~S}$ head and body. $\mathrm{d}-\mathrm{f}$ ) Structural view from the subunit interface of the 70S ribosomes bound with tRNAs and EF-G, in which $50 \mathrm{~S}$ and $30 \mathrm{~S}$ are shown in blue and yellow with transparency, tRNAs in solid orange and green, EF-G in solid red. g-i) Intersubunit rotation accompanying translocation viewed from the solvent side of the $30 \mathrm{~S}$. The $50 \mathrm{~S}$ and $30 \mathrm{~S}$ are displayed in blue and yellow, and the counter-clockwise rotation of $30 \mathrm{~S}$ relative to $50 \mathrm{~S}$ is indicated by the arrows. Degrees of intersubunit rotation (from [17]) measured relative to the structure of nonrotated ribosome (PDBID 4V51). a, d, g) The rotated (R) pre-translocation ribosome is bound with EF-G and tRNAs in A/P and P/E hybrid states (PDBID 4V7D). b, e, h) The partially rotated ribosome containing tRNAs in ap/P and pe/E tRNAs chimeric (CH) states (PDBID 4W29). c, f, i) The post-translocation EF-G-bound ribosome in nonrotated (NR) conformation (PDBID 4V5F), which contains tRNAs bound in classical $\mathrm{P} / \mathrm{P}$ and $\mathrm{E} / \mathrm{E}$ state.

logues of GTP trap EF-G on the ribosome. These results suggest that GTP hydrolysis is not required for translocation but it is essential for EF-G release. Consistent with the Spirin-Kaziro experiments, more recent kinetic studies have shown that the non-hydrolysable analogues of GTP do not alter the translocation pathway [26, 27]. Furthermore, replacing GTP with non-hydrolysable analogues only moderately affects the rate of a single round of translocation, reducing it by $2-50$ folds depending on experimental conditions [19, 26, 28, 29].

Another fundamental aspect of the translocation mechanism was discovered by Spirin and his colleagues in the experiments demonstrating that tRNAs can translocate through the ribosome in the absence of mRNA $[10,11]$. These observations suggest that the movement of mRNA is driven by the translocation of the associated anti-codon stem-loops (ASLs) of A- and P-site tRNAs. More recent studies indicate that mRNA translocation requires the presence of ASL in the A site and full-length tRNA in the P site of the pre-translocation ribosome [30]. Consistent with the idea of tRNA-driven translocation of mRNA, single-molecule measurements showed that mRNA translocates three nucleotides at a time without detectable sub-steps [31]. Since tRNAs interact with both 30S and 50S subunits (Fig. 1, a-f), the tRNA-driven mechanism of translocation indicates that both ribosomal subunits are involved in this process.

\section{STRUCTURAL REARRANGEMENTS OF THE RIBOSOME: THE LOCKING- UNLOCKING HYPOTHESIS}

In 1968-1969, Spirin proposed the locking-unlocking model of translocation based on the subunit organization of ribosome structure $[1,2,6]$. This model postulat- 
ed that (i) tRNA translocation involves transition from the "locked" to the "unlocked" ribosome conformation that facilitates tRNA diffusion through the ribosome; (ii) peptidyl-transferase reaction triggers formation of an intermediate of translocation in which tRNAs are shifted on the large subunit but not yet translocated on the small subunit; (iii) translocation involves movement of the ribosomal subunits relative to each other. Similar ideas were independently proposed by M. S. Bretscher [32]. As discussed below, many aspects of this model turned out to be prophetic. To this day, the rate limiting step of translocation is often referred to as "unlocking".

Neutron scattering experiments performed by Spirin, Serdyuk, and May provided an early indirect evidence for intersubunit rearrangements accompanying translocation [33, 34]. Nevertheless, further verification of the key predictions of the locking-unlocking model took several decades and required developing new experimental approaches such as chemical probing of RNA structure, cryogenic electron microscopy (cryo-EM), and single-molecule Förster resonance energy transfer (smFRET) microscopy [35].
Twenty years after the introduction of the locking-unlocking model, Danesh Moazed and Harry Noller used chemical probing for mapping of the tRNA binding sites to demonstrate that the reaction of transpeptidation triggers spontaneous translocation of the acceptors stems of the resulting peptidyl- and deacylated tRNAs from the A and P to P and E sites of the large subunit, respectively, while tRNA ASLs remain in the original $A$ and $P$ sites of the small subunit [36]. Hence, intermediate $\mathrm{A} / \mathrm{P}$ and $\mathrm{P} / \mathrm{E}$ hybrid states of tRNA binding were formed (Fig. 1, a and d). Completion of translocation of tRNAs on the small subunit was shown to require EF-G and GTP (Fig. 1, a-f).

A decade later, another key prediction of the locking-unlocking model was corroborated by cryo-EM reconstruction of the EF-G-ribosome complex performed by Joachim Frank and Rajendra Agrawal [37]. These experiments demonstrated that EF-G binding induces rotation of the small $30 \mathrm{~S}$ subunit relative to the large 50S subunit parallel to the plane of the intersubunit interface (Fig. 1, g-i; Fig. 2, a and b). The discoveries of the hybrid-state intermediate and intersubunit rotation

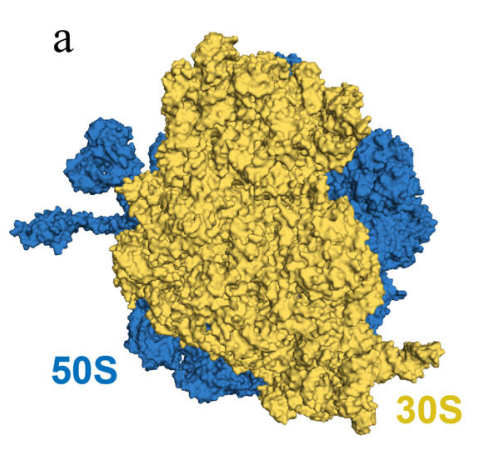

NR 70S

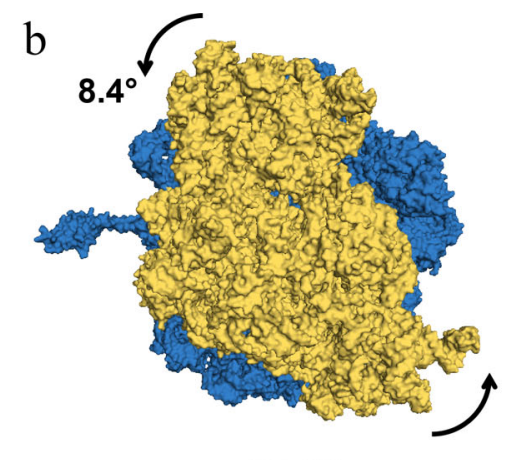

d

R 70S

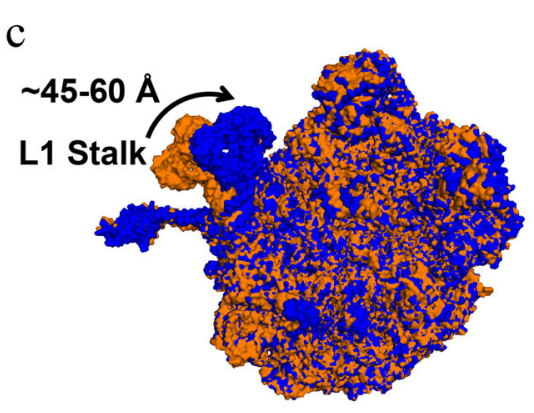

NR/R 50S

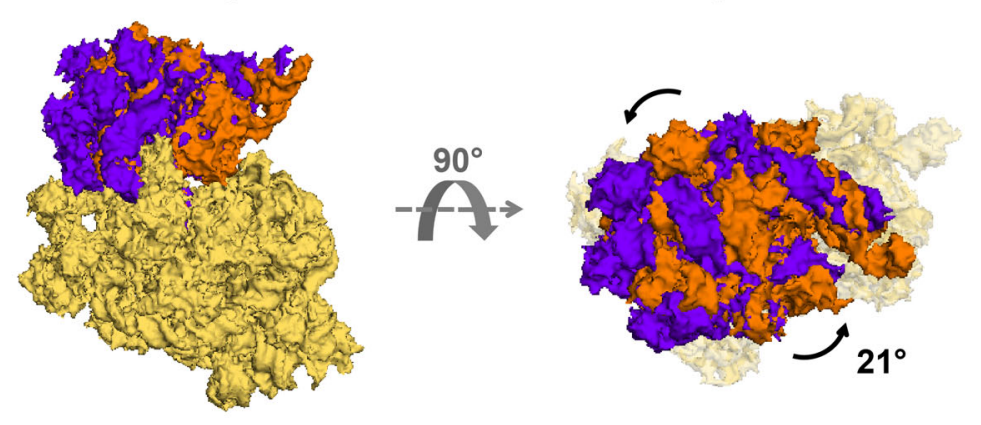

NR/CH 30 S Head

Fig. 2. Intersubunit rotation, L1 stalk movement and $30 \mathrm{~S}$ head swivel observed in EF-G-free ribosomes. Structures of the $70 \mathrm{~S}$ ribosometRNA complex (PDBID 4V9D) in the non-rotated (NR), classical state (a) and in the rotated (R), hybrid state (b). 50S and 30S ribosomal subunits are shown in blue and yellow, respectively. Both ribosome structures are viewed from the solvent side of the $30 \mathrm{~S}$ subunit. The counter-clockwise rotation of $30 \mathrm{~S}$ relative to $50 \mathrm{~S}$ is indicated by the curved arrows. c) The L1 stalk in 50S subunits of NR (orange) and R (blue) ribosomes (PDBID 4V9D) superimposed by structural alignment of the 23S rRNA. d and e) The swiveling motion of the $30 \mathrm{~S}$ head domain is shown by structural alignment of the body and platform domains (yellow) of the 16S rRNAs of NR (PDBID 4V51) and chimeric state (CH) (PDBID 4W29) ribosomes. The 30S is viewed from the solvent side of the $30 \mathrm{~S}$ subunit (d) and from the "top" of the $30 \mathrm{~S}$ head domain (e). The NR and CH 30S head domains are shown in orange and purple, respectively. Degrees of 30S head swivel in CH and intersubunit rotation in $\mathrm{R}$ (from [17]) are measured relative to the NR 70S (PDBID 4V51). 
were followed by numerous structural and single-molecule studies that provided unprecedented insights into the structural rearrangements of the ribosome, tRNAs, and EF-G accompanying translocation [38, 39].

Cryo-EM and FRET experiments revealed that 6$10^{\circ}$ intersubunit rotation is coupled with the movement of peptidyl- and deacylated tRNAs into $\mathrm{A} / \mathrm{P}$ and $\mathrm{P} / \mathrm{E}$ hybrid states (Fig. 2, a and b) [38, 40-43]. These data established equivalence of the nonrotated and rotated conformations with classical and hybrid states of tRNA binding, respectively. Cryo-EM and smFRET studies have also shown that formation of the rotated, hybrid state of the ribosome is accompanied by the inward movement of the mobile domain of the large ribosomal subunit named L1 stalk (Fig. 2c), which comprises ribosomal protein $\mathrm{uL1}$ and helices 76, 77, and 78 of the 23S rRNA [35, 39]. Upon transition from the open to closed conformation, the extremity of the L1 stalk moves by as much as $60 \AA$ and the L1 stalk becomes bound to the elbow of the P/E tRNA.

smFRET studies also demonstrated that in the absence of EF-G, the pretranslocation ribosome spontaneously fluctuates between the nonrotated, classical and the rotated, hybrid state conformations (Fig. 2, a and b) [38, 44-46]. Binding of EF-G $\cdot$ GTP transiently stabilizes the rotated, hybrid state conformation (Fig. 1, a, d, and g); translocation of mRNA and tRNA on the small subunit is coupled with the reverse transition into non-rotated, classical state conformation (Fig. 1, c, f, and i) [26, 40, 47].

In addition to intersubunit rearrangements and movement of the L1 stalk, translocation is accompanied by large structural changes within the small ribosomal subunit. The small subunit comprises three structural domains: head, body, and platform. Structural studies show that the $30 \mathrm{~S}$ head rotates by up to $20^{\circ}$ relative to the rest of the small subunit around the axis that is orthogonal to the axis of intersubunit rotation (Fig. 2, d and e) $[48,49]$. In the EF-G-bound intermediate of translocation visualized by $\mathrm{X}$-ray crystallography and cryo-EM, in which the $30 \mathrm{~S}$ head is observed in a swiveled conformation, two tRNAs are translocated along the 50S subunit and the 30S platform/body but not yet translocated relative to the $30 \mathrm{~S}$ head (Fig. 1, b, e, and h) [50, 51]. In these positions named ap/P and pe/E chimeric states, tRNAs are trapped midway between hybrid $(\mathrm{A} / \mathrm{P}$ and $\mathrm{P} / \mathrm{E})$ and posttranslocation classical (P/P and E/E states) and likely represent a late intermediate of translocation.

The tip of domain IV of EF-G plays a critical role in translocation activity of EF-G and reading frame maintenance [19, 52-54]. When EF-G is bound in the rotated hybrid state conformation of the pretranslocation ribosome, domain IV of EF-G is positioned next to ASL of A/P tRNA (Fig. 1, a and d) [55]. Upon translocation, domain IV of EF-G docks into the A site of the small subunit vacated by the peptidyl-tRNA (Fig. 1, c and f) $[38,56]$. Hence, upon reverse intersubunit rotation and
30S back-swivel, domain IV of EF-G displaces ASL of peptidyl-tRNA and prevents its backward movement.

Which of the aforementioned conformational rearrangements is the rate-limiting step that "unlocks" the ribosome (using Spirin's terminology) and facilitates tRNA translocation is not entirely clear. Reaction of transpeptidation "unlocks" the ribosome in a sense that it enables spontaneous intersubunit rotation and fluctuations of tRNAs between the classical and hybrid states [44-46]. However, in the absence of EF-G, these fluctuations are unproductive and do not lead to tRNA/mRNA translocation on the small subunit [4446]. Several lines of evidence suggest that the domain IV of EF-G destabilizes interactions of A-site tRNA with 16S rRNA [57-61]. Hence, the EF-G-induced changes in the A site may "unlock" the ribosome. Finally, another possible "unlocking" rearrangement is swiveling of the 30S head, which opens the path for tRNA movement from $\mathrm{P}$ to E site that is otherwise constricted [49]. Further studies are needed to establish complete sequence of the structural rearrangements accompanying translocation and identify the rate-limiting step in this process.

\section{ENERGETICS OF TRANSLOCATION AND BROWNIAN RATCHET MODEL}

Although translation is greatly accelerated by EF-G, the Spirin and Pestka laboratories demonstrated that translocation can occur spontaneously in the absence of protein factors [7, 8, 62]. It was found in the Spirin laboratory that spontaneous translocation is stimulated by modification of the universally conserved ribosomal protein (u)S12 of the $30 \mathrm{~S}$ subunit by thiol-specific reagents, which were added to inactivate EF-G and thus rule out the presence of trace amounts of this protein [7, 63, 64]. More recent studies indicated that removal of the $30 \mathrm{~S}$ proteins uS12 and uS13 enhanced the factor-free translocation possibly by weakening tRNA interactions with the 30S A and P sites, respectively [65]. Furthermore, it was reported that a single-round factor-free translocation could be induced by antibiotics sparsomycin, lincomycin, and chloramphenicol that bind to the 50S A site and thus destabilize the A-site tRNA binding [20, 66].

Based on the observations of factor-free translocation, Spirin postulated that translocation is an inherent property of the ribosome and that energy of the peptidyltransfer reactions is sufficient to promote tRNA movement [3]. However, the slow rate of spontaneous translocation and the observation of reverse spontaneous translocation in some tRNA/mRNA contexts suggest that the reaction of transpeptidation is not the only energy source of translocation, which is also promoted by the energy stored in EF-G•GTP. Indeed, it was estimated that transpeptidation-driven translocation would require $\sim 80 \%$ efficiency of the conversion of chemical energy 
into mechanical motion [67]. Such high efficiency is untypical for macromolecular motors [67].

Two alternative idealized models, the power stroke and the Brownian ratchet models, are employed to describe conversion of chemical energy into mechanical work by macromolecular motors [68, 69]. Chemical energy may be converted into elastic energy or conformational transition that drives large conformational change of the macromolecule, i.e., the power stroke. Alternatively, energy of chemical reaction may be used to bias random, thermally-driven motions of the macromolecule into unidirectional movement. Thus, chemical reaction plays a role that is similar to a pawl directing the movement of a wheel of mechanical ratchet. The chemical change either strictly precedes conformational change (the power stroke) or follows it (the Brownian ratchet) [68]. These two mechanisms can be distinguished by examining the load dependence of the molecular motor movement [68].

Several groups hypothesized that tRNA translocation is mediated by the power stroke of domain IV of EFG driven by GTP hydrolysis [19, 70, 71]. This hypothesis is supported by kinetic data suggesting that GTP hydrolysis by EF-G precedes translocation [19]. However, the Spirin-Kaziro experiments and more recent kinetic measurements show that translocation occurs rapidly and efficiently in the absence of GTP hydrolysis, when GTP is replaced with non-hydrolysable analogues [9, 19, 2326, 28, 29]. These data suggest that GTP hydrolysis by EF-G is not coupled with translocation and argue against the GTP hydrolysis-driven power stroke.

Based on the observations of spontaneous, factorfree translocation, Spirin reasoned that Brownian motions of tRNA are sufficient to explain translocation without invoking the power stroke by EF-G [3, 12, 13]. This hypothesis was further corroborated by the smFRET data demonstrating spontaneous intersubunit rotation and fluctuations of tRNA between the classical and hybrid states [44-46]. Spirin's ideas were ultimately reinforced by the single-molecule optical tweezer measurements of ribosome translocation against applied force showing that EF-G-catalyzed translocation is also best described by the Brownian ratchet model [67].

In the Brownian ratchet mechanism of EF-G-catalyzed translocation, EF-G•GTP likely acts as a pawl of the ratchet that biases tRNA diffusion through the ribosome and couples translocation with the ribosome dynamics $[14,15]$. The structure of EF-G trapped in the rotated pretranslocation ribosome (Fig. 1, a and d) reveals the basis for coupling of intersubunit rotation and tRNA/mRNA translocation [55]. Unproductive spontaneous fluctuations of the ribosome from the rotated into nonrotated conformation leads to the return of peptidyl-tRNA from the hybrid A/P into the classical A/A state. However, when EF-G is bound to the rotated pretranslocation ribosome, domain IV of EF-G creates steric hindrance for the return of peptidyl-tRNA from the hybrid A/P into classical A/A state [55]. Similarly, domain IV of EF-G sterically blocks the return of peptidyl-tRNA from the chimeric ap/P into the classical A/A state upon back-swivel of the $30 \mathrm{~S}$ head [72]. Furthermore, upon translocation of peptidyltRNA from the A to P site of the small subunit, domain IV of EF-G docks into the 30S A site thus rendering the tRNA movement irreversible (Fig. 1, c and f).

\section{RIBOSOME TRANSLOCATION IN REGULATION OF TRANSLATION: RIBOSOME AS A HELICASE}

While unprecedented molecular details of the translocation mechanism have recently emerged from the structural studies and single-molecule biophysical measurements, it remains less clear how the rate of translocation is modulated in live cells to regulate translation elongation. Eukaryotic translocase EF-2 was shown to be downregulated under stress conditions by phosphorylation [73-75]. Besides, EF-2 can be inactivated by ADPribosylation catalyzed by diphtheria toxin [76]. A number of antibiotics hamper cell growth by hindering translocation in bacteria [14]. Arguably the least understood and most fascinating aspect of the translocation regulation is modulation of the translocation rate by mRNA secondary structure.

Computational analyses suggest that most, if not all, mRNAs have the propensity to form extensive intramolecular secondary structures throughout the entire sequence including the Open Reading Frame (ORF) [77]. mRNA folding results in the formation of compact structures with short end-to-end distances [78]. In vivo transcriptome-wide RNA structure probing studies [7984] show that mRNAs fold in live cells, at least to some degree, despite the presence of RNA helicases and other RNA binding proteins, which can disrupt RNA secondary structure. Consistent with the idea that mRNAs form extensive secondary structure in vivo, a number of structured mRNA elements were shown to regulate translation initiation, including bacterial riboswitches [85], frameshift-inducing hairpins and pseudoknots of eukaryotic viruses [86], Internal Ribosome Entry Sites (IRES) [87], Iron Response Elements (IRE) in the 5'UTR of transcripts coding for proteins involved in iron metabolism [88], and Cap-Independent Translational Enhancers (CITEs) [89]. Furthermore, protein and miRNA binding to mRNA was found to be governed by the RNA structure, which can occlude sites [90-95] providing further evidence for the importance of mRNA secondary structure.

Biochemical and single-molecule experiments revealed that the translating ribosome is a very efficient helicase [96, 97], which unwinds three base pairs per translocation step (Fig. 3). The translating ribosome unfolds mRNA secondary structure because the narrow mRNA channel of the small ribosomal subunit can only 


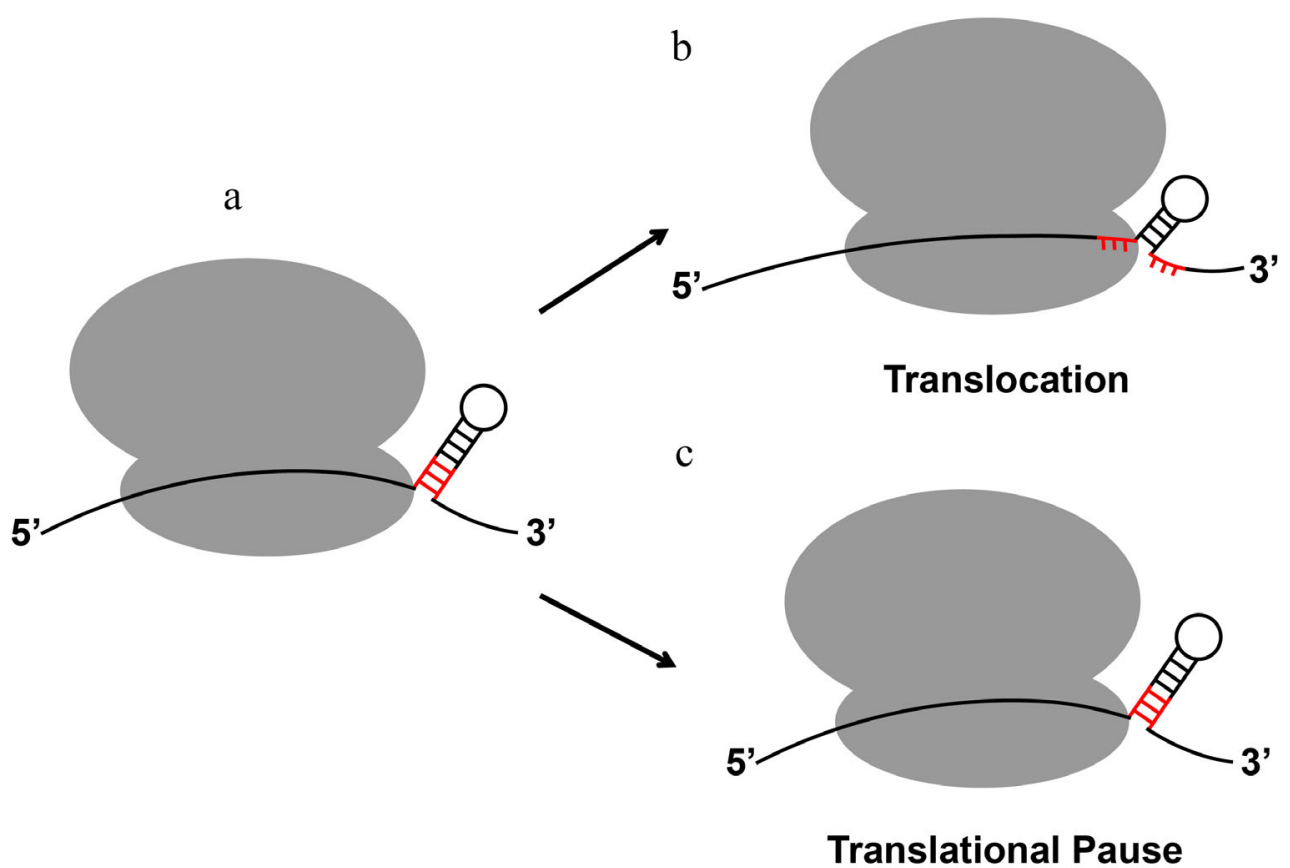

Fig. 3. mRNA stem-loops can either be quickly unwound by the ribosome or induce ribosome pausing. When positioned at the mRNA entry channel, certain regulatory secondary structures of mRNA induce translational pause despite the helicase activity of the elongating ribosome. The three base pairs of the stem-loop adjacent to the mRNA entry channel that become unwound by a translocation step are shown in red.

accommodate a single-stranded mRNA [96, 98-101]. Consistent with the demonstrations of helicase activity of the ribosome, transcriptome-wide ribosome profiling analysis demonstrates that most of the secondary structure elements within the coding regions of mRNAs do not influence the rate of translation elongation [102]. Most structured mRNA elements, which regulate translation, reside either in the $5^{\prime}$ - or $3^{\prime}$-UTRs.

Helicase activity of the ribosome likely plays a major role in the remodeling of mRNA structure and regulating mRNA interactions with RNA-binding proteins [103]. For example, mRNA translation in poly-ribosomes renders the mRNA ORF devoid of secondary structure due to the ribosome helicase activity [103-105]. The pioneer round of mRNA translation by the ribosome, which displaces exon junction protein complexes (EJCs) and other proteins deposited on mRNA in the nucleus [106], may enable mRNA folding into compact structures after termination of protein synthesis. Indeed, the single-molecule-resolution fluorescent in situ hybridization (smFISH) and proximity-ligation studies indicate lack of interactions between the distant segments of nuclear mRNAs bound with exon junction protein complexes (EJCs) [104, 107]. By contrast, the 5'- and 3 '-ends of cytoplasmic mRNAs, which are not actively translated, are co-localized through the formation of intramolecular secondary structure $[104,105]$ that tend to bring mRNA ends in close proximity [78, 108].

Paradoxically, in spite of the ribosome helicase activity, certain RNA stem-loop structures can induce ribo- some stalling that results in accumulation of truncated polypeptides [109] and No-Go mRNA decay (Fig. 3) [110]. Furthermore, the evolutionarily conserved mRNA stem-loops and pseudoknots trigger programmed translation pauses [111] and stimulate -1 programmed ribosomal frameshifting (PRF), which controls expression of a number of proteins in bacteria, viruses and eukaryotes [112]. In particular, -1 PRF regulates synthesis of DNA polymerase III in bacteria [113]; HIV cytokine receptor ccr5 in higher eukaryotes [114]; gag-pol proteins in retroviruses, including Human Immunodeficiency Virus (HIV) [115]; and C-terminally extended polyprotein in coronaviruses, including SARS-CoV-2, which caused the COVID-19 pandemic [116, 117].

The mechanism of ribosome pausing induced by mRNA secondary structure is not fully understood. A number of published single-molecule studies suggest that slow unwinding of a secondary structure, to which ribosome pausing is often attributed, is an unlikely explanation of the extent of translation inhibition induced by certain mRNA stem-loops [31, 118, 119]. Translocation through three GC base pairs is only 2 to 3 -fold slower than translocation along a single-stranded codon $[31,118,119]$ indicating that the stability of the three base pairs adjacent to the mRNA channel has a relatively moderate effect on translocation rate. By contrast, the frameshift-inducing stem-loops and pseudoknots were shown to produce extended ribosome pauses [120-127].

It appears that rather than creating a simple road block for the ribosome, mRNA stem-loops induce pro- 
grammed ribosome pauses by making specific interactions with the ribosome. Recent studies suggest that the frameshift-inducing mRNA stem-loops can perturb translation elongation by docking into the $30 \mathrm{~S}$ A site hindering tRNA binding [128]. Furthermore, when positioned at the entry of $30 \mathrm{~S}$ mRNA channel, frameshiftinducing stem-loops and pseudoknots were shown to inhibit the rate of ribosomal translocation by more than one order of magnitude in a number of kinetic ensemble and single-molecule experiments [120, 121, 126-129]. It has been recently demonstrated that upon encountering the mRNA secondary structure the ribosome translocates through two alternative pathways (fast and slow) [31]. Interactions of the frameshift-inducing stem-loops and pseudoknots with the mRNA entry channel may increase the flux through the slow pathway and thus decrease the average rate of ribosome translocation [31].

Many of Spirin's ingenious insights into the mechanism of ribosomal translocation were corroborated in the last few decades with advances brought by high-resolution structures of the ribosome and single-molecule biophysical experiments. Nevertheless, ribosomal translocation along mRNA remains one of the most fascinating steps of proteins synthesis. The complete "movie" reconstructing structural rearrangements of the ribosome, EF$\mathrm{G}$, and tRNA during translocation is yet to materialize. A more complete understanding of how ribosome translocation remodels mRNA secondary structure and modulates interactions of mRNA with many regulatory proteins is just beginning to emerge. Mechanisms of regulation of ribosome translocation by mRNA secondary structure await further investigation.

Acknowledgments. This article is dedicated to the memory of Prof. Spirin who was undergraduate research mentor to one of the authors (D. N. E.). D. N. E. is grateful to Alexander Sergeevich Spirin for his invaluable lessons of critical thinking and research rigor. The authors thank Gregory Ballard for his comments on the manuscript.

Funding. The research in the Ermolenko laboratory is supported by NIH grants R01GM099719 and R01GM132041.

Ethics declarations. The authors declare no conflicts of interest in financial or any other sphere. This article does not contain any studies involving human participants or animals performed by any of the authors.

\section{REFERENCES}

1. Spirin, A. S. (1968) How does the ribosome work? A hypothesis based on the two subunit construction of the ribosome, Curr. Mod. Biol., 2, 115-127, doi: 10.1016/03032647(68)90017-8.

2. Spirin, A. S. (1969) A model of the functioning ribosome: locking and unlocking of the ribosome subparticles, Cold
Spring Harb. Symp. Quant. Biol., 34, 197-207, doi: 10.1101/sqb.1969.034.01.026.

3. Spirin, A. S. (1985) Ribosomal translocation: facts and models, Prog. Nucleic Acid. Res. Mol. Biol., 32, 75-114, doi: 10.1016/s0079-6603(08)60346-3.

4. Spirin, A. S. (2009) The ribosome as a conveying thermal ratchet machine, J. Biol. Chem., 284, 21103-21119, doi: 10.1074/jbc.X109.001552.

5. Finkelstein, A. V., Razin, S. V., and Spirin, A. S. (2018) Intersubunit mobility of the ribosome, Mol. Biol. (Mosk.), 52, 921-934, doi: 10.1134/S0026898418060083.

6. Spirin, A. S. (1968) On the mechanism of ribosome function. The hypothesis of locking-unlocking of subparticles [in Russsian], Dokl. Akad. Nauk SSSR, 179, 1467-1470.

7. Gavrilova, L. P., and Spirin, A. S. (1971) Stimulation of "non-enzymic" translocation in ribosomes by pchloromercuribenzoate, FEBS Lett., 17, 324-326, doi: 10.1016/0014-5793(71)80177-1.

8. Gavrilova, L. P., and Spirin, A. S. (1974) "Nonenzymatic" translation, Methods Enzymol., 30, 452-462, doi: 10.1016/ 0076-6879(74)30045-6.

9. Belitsina, N. V., Glukhova, M. A., and Spirin, A. S. (1975) Translocation in ribosomes by attachment-detachment of elongation factor $\mathrm{G}$ without GTP cleavage: evidence from a column-bound ribosome system, FEBS Lett., 54, 35-38, doi: 10.1016/0014-5793(75)81062-3.

10. Belitsina, N. V., Tnalina, G. Z., and Spirin, A. S. (1981) Template-free ribosomal synthesis of polylysine from lysyltRNA, FEBS Lett., 131, 289-292, doi: 10.1016/00145793(81)80387-0.

11. Belitsina, N. V., Tnalina, G. Z., and Spirin, A. S. (1982) Template-free ribosomal synthesis of polypeptides from aminoacyl-tRNAs, Biosystems, 15, 233-241, doi: 10.1016/ 0303-2647(82)90008-9.

12. Spirin, A. S. (2002) Ribosome as a molecular machine, FEBS Lett., 514, 2-10, doi: 10.1016/s0014-5793(02)02309-8.

13. Spirin, A. S. (2004) The ribosome as an RNA-based molecular machine, RNA Biol., 1, 3-9, doi: 10.4161/rna.1.1.889.

14. Ling, C., and Ermolenko, D. N. (2016) Structural insights into ribosome translocation, Wiley Interdiscip. Rev. $R N A, 7$, 620-636, doi: 10.1002/wrna.1354.

15. Noller, H. F., Lancaster, L., Zhou, J., and Mohan, S. (2017) The ribosome moves: RNA mechanics and translocation, Nat. Struct. Mol. Biol., 24, 1021-1027, doi: 10.1038/ nsmb.3505.

16. Rodnina, M. V., Peske, F., Peng, B. Z., Belardinelli, R., and Wintermeyer, W. (2019) Converting GTP hydrolysis into motion: versatile translational elongation factor $G$, Biol. Chem., 401, 131-142, doi: 10.1515/hsz-2019-0313.

17. Mohan, S., Donohue, J. P., and Noller, H. F. (2014) Molecular mechanics of $30 \mathrm{~S}$ subunit head rotation, Proc. Natl. Acad. Sci. USA, 111, 13325-13330, doi: 10.1073/pnas. 1413731111.

18. Gavrilova, L. P., Kostiashkina, O. E., Koteliansky, V. E., Rutkevitch, N. M., and Spirin, A. S. (1976) Factor-free ("non-enzymic") and factor-dependent systems of translation of polyuridylic acid by Escherichia coli ribosomes, J. Mol. Biol., 101, 537-552, doi: 10.1016/0022-2836(76)90243-6.

19. Rodnina, M. V., Savelsbergh, A., Katunin, V. I., and Wintermeyer, W. (1997) Hydrolysis of GTP by elongation factor $\mathrm{G}$ drives tRNA movement on the ribosome, Nature, 385, 37-41, doi: 10.1038/385037a0. 
20. Fredrick, K., and Noller, H. F. (2003) Catalysis of ribosomal translocation by sparsomycin, Science, 300, 11591162, doi: 10.1126/science. 1084571 .

21. Parmeggiani, A., and Sander, G. (1981) Properties and regulation of the GTPase activities of elongation factors $\mathrm{Tu}$ and G, and of initiation factor 2, Mol. Cell. Biochem., 35, 129-158, doi: 10.1007/BF02357085.

22. Moazed, D., Robertson, J. M., and Noller, H. F. (1988) Interaction of elongation factors EF-G and EF-Tu with a conserved loop in 23S RNA, Nature, 334, 362-364, doi: $10.1038 / 334362 \mathrm{a} 0$.

23. Inoue-Yokosawa, N., Ishikawa, C., and Kaziro, Y. (1974) The role of guanosine triphosphate in translocation reaction catalyzed by elongation factor G, J. Biol. Chem., 249, 4321-4323, doi: 10.1016/S0021-9258(19)42519-2.

24. Belitsina, N. V., Glukhova, M. A., and Spirin, A. S. (1976) Stepwise elongation factor G-promoted elongation of polypeptides on the ribosome without GTP cleavage, J. Mol. Biol., 108, 609-613, doi: 10.1016/s00222836(76)80140-4.

25. Belitsina, N. V., Glukhova, M. A., and Spirin, A. S. (1979) Elongation factor G-promoted translocation and polypeptide elongation in ribosomes without GTP cleavage: use of columns with matrix-bound polyuridylic acid, Methods Enzymol., 60, 761-779, doi: 10.1016/s0076-6879(79)60070-8.

26. Ermolenko, D. N., and Noller, H. F. (2011) mRNA translocation occurs during the second step of ribosomal intersubunit rotation, Nat. Struct. Mol. Biol., 18, 457-462, doi: $10.1038 / \mathrm{nsmb} .2011$.

27. Flis, J., Holm, M., Rundlet, E. J., Loerke, J., Hilal, T., et al. (2018) tRNA Translocation by the eukaryotic $80 \mathrm{~S}$ ribosome and the impact of GTP hydrolysis, Cell Rep., 25, 2676-2688.e7, doi: 10.1016/j.celrep.2018.11.040.

28. Pan, D., Kirillov, S. V., and Cooperman, B. S. (2007) Kinetically competent intermediates in the translocation step of protein synthesis, Mol. Cell, 25, 519-529, doi: 10.1016/j.molcel.2007.01.014.

29. Salsi, E., Farah, E., and Ermolenko, D. N. (2016) EF-G activation by phosphate analogs, J. Mol. Biol., 428, 22482258, doi: $10.1016 /$ j.jmb.2016.03.032.

30. Joseph, S., and Noller, H. F. (1998) EF-G-catalyzed translocation of anticodon stem-loop analogs of transfer RNA in the ribosome, EMBO J., 17, 3478-3483, doi: 10.1093/emboj/17.12.3478.

31. Desai, V. P., Frank, F., Lee, A., Righini, M., Lancaster, L., et al. (2019) Co-temporal force and fluorescence measurements reveal a ribosomal gear shift mechanism of translation regulation by structured mRNAs, Mol. Cell, 75, 10071019.e5, doi: 10.1016/j.molcel.2019.07.024.

32. Bretscher, M. S. (1968) Translocation in protein synthesis: a hybrid structure model, Nature, 218, 675-677, doi: $10.1038 / 218675 \mathrm{a} 0$.

33. Spirin, A. S., Baranov, V. I., Polubesov, G. S., Serdyuk, I. N., and May, R. P. (1987) Translocation makes the ribosome less compact, J. Mol. Biol., 194, 119-126, doi: 10.1016/0022-2836(87)90720-0.

34. Serdyuk, I., Baranov, V., Tsalkova, T., Gulyamova, D., Pavlov, M., et al. (1992) Structural dynamics of translating ribosomes, Biochimie, 74, 299-306, doi: 10.1016/03009084(92)90107-p.

35. Frank, J., and Gonzalez, R. L., Jr. (2010) Structure and dynamics of a processive Brownian motor: the translating ribosome, Annu. Rev. Biochem., 79, 381-412, doi: 10.1146/ annurev-biochem-060408-173330.

36. Moazed, D., and Noller, H. F. (1989) Intermediate states in the movement of transfer RNA in the ribosome, Nature, 342, 142-148, doi: 10.1038/342142a0.

37. Frank, J., and Agrawal, R. K. (2000) A ratchet-like intersubunit reorganization of the ribosome during translocation, Nature, 406, 318-322, doi: 10.1038/35018597.

38. Valle, M., Zavialov, A., Sengupta, J., Rawat, U., Ehrenberg, M., and Frank, J. (2003) Locking and unlocking of ribosomal motions, Cell, 114, 123-134, doi: 10.1016/S0092-8674(03)00476-8.

39. Korostelev, A., Ermolenko, D. N., and Noller, H. F. (2008) Structural dynamics of the ribosome, Curr. Opin. Chem. Biol., 12, 674-683, doi: 10.1016/j.cbpa.2008.08.037.

40. Ermolenko, D. N., Majumdar, Z. K., Hickerson, R. P., Spiegel, P. C., Clegg, R. M., and Noller, H. F. (2007) Observation of intersubunit movement of the ribosome in solution using FRET, J. Mol. Biol., 370, 530-540, doi: 10.1016/j.jmb.2007.04.042.

41. Ermolenko, D. N., Spiegel, P. C., Majumdar, Z. K., Hickerson, R. P., Clegg, R. M., and Noller, H. F. (2007) The antibiotic viomycin traps the ribosome in an intermediate state of translocation, Nat. Struct. Mol. Biol., 14, 493497, doi: $10.1038 / \mathrm{nsmb} 1243$.

42. Agirrezabala, X., Lei, J., Brunelle, J. L., Ortiz-Meoz, R. F., Green, R., and Frank, J. (2008) Visualization of the hybrid state of tRNA binding promoted by spontaneous ratcheting of the ribosome, Mol. Cell, 32, 190-197, doi: 10.1016/ j.molcel.2008.10.001.

43. Julian, P., Konevega, A. L., Scheres, S. H., Lazaro, M., Gil, D., et al. (2008) Structure of ratcheted ribosomes with tRNAs in hybrid states, Proc. Natl. Acad. Sci. USA, 105, 16924-16927, doi: 10.1073/pnas.0809587105.

44. Blanchard, S. C., Kim, H. D., Gonzalez, R. L., Jr., Puglisi, J. D., and Chu, S. (2004) tRNA dynamics on the ribosome during translation, Proc. Natl. Acad. Sci. USA, 101, 1289312898, doi: 10.1073/pnas.0403884101.

45. Cornish, P. V., Ermolenko, D. N., Noller, H. F., and Ha, T. (2008) Spontaneous intersubunit rotation in single ribosomes, Mol. Cell, 30, 578-588, doi: 10.1016/j.molcel. 2008.05.004.

46. Fei, J., Kosuri, P., MacDougall, D. D., and Gonzalez, R. L., Jr. (2008) Coupling of ribosomal L1 stalk and tRNA dynamics during translation elongation, Mol. Cell, 30, 348359, doi: 10.1016/j.molcel.2008.03.012.

47. Marshall, R. A., Aitken, C. E., and Puglisi, J. D. (2009) GTP hydrolysis by IF2 guides progression of the ribosome into elongation, Mol. Cell, 35, 37-47, doi: 10.1016/j.molcel. 2009.06.008.

48. Spahn, C. M., Gomez-Lorenzo, M. G., Grassucci, R. A., Jorgensen, R., Andersen, G. R., et al. (2004) Domain movements of elongation factor eEF2 and the eukaryotic 80S ribosome facilitate tRNA translocation, EMBO J., 23, 1008-1019, doi: 10.1038/sj.emboj.7600102.

49. Schuwirth, B. S., Borovinskaya, M. A., Hau, C. W., Zhang, W., Vila-Sanjurjo, A., et al. (2005) Structures of the bacterial ribosome at $3.5 \AA$ resolution, Science, 310, 827834, doi: 10.1126/science.1117230.

50. Ramrath, D. J., Lancaster, L., Sprink, T., Mielke, T., Loerke, J., et al. (2013) Visualization of two transfer RNAs trapped in transit during elongation factor G-mediated 
translocation, Proc. Natl. Acad. Sci. USA, 110, 2096420969, doi: 10.1073/pnas.1320387110.

51. Zhou, J., Lancaster, L., Donohue, J. P., and Noller, H. F. (2014) How the ribosome hands the A-site tRNA to the P site during EF-G-catalyzed translocation, Science, 345, 1188-1191, doi: 10.1126/science. 1255030 .

52. Martemyanov, K. A., and Gudkov, A. T. (1999) Domain IV of elongation factor $\mathrm{G}$ from Thermus thermophilus is strictly required for translocation, FEBS Lett., 452, 155159, doi: 10.1016/S0014-5793(99)00635-3.

53. Niblett, D., Nelson, C., Leung, C. S., Rexroad, G., Cozy, J., et al. (2021) Mutations in domain IV of elongation factor EF-G confer-1 frameshifting, $R N A, 27,40-53$, doi: 10.1261/rna.077339.120.

54. Peng, B. Z., Bock, L. V., Belardinelli, R., Peske, F., Grubmuller, H., and Rodnina, M. V. (2019) Active role of elongation factor $\mathrm{G}$ in maintaining the mRNA reading frame during translation, $S c i$. $A d v$., 5, eaax8030, doi: 10.1126/sciadv.aax8030.

55. Brilot, A. F., Korostelev, A. A., Ermolenko, D. N., and Grigorieff, N. (2013) Structure of the ribosome with elongation factor $\mathrm{G}$ trapped in the pretranslocation state, Proc. Natl. Acad. Sci. USA, 110, 20994-20999, doi: 10.1073/ pnas. 1311423110.

56. Salsi, E., Farah, E., Dann, J., and Ermolenko, D. N. (2014) Following movement of domain IV of elongation factor $\mathrm{G}$ during ribosomal translocation, Proc. Natl. Acad. Sci. USA, 111, 15060-15065, doi: 10.1073/pnas.1410873111.

57. Gao, Y. G., Selmer, M., Dunham, C. M., Weixlbaumer, A., Kelley, A. C., and Ramakrishnan, V. (2009) The structure of the ribosome with elongation factor $\mathrm{G}$ trapped in the posttranslocational state, Science, 326, 694-699, doi: $10.1126 /$ science. 1179709 .

58. Khade, P. K., and Joseph, S. (2011) Messenger RNA interactions in the decoding center control the rate of translocation, Nat. Struct. Mol. Biol., 18, 1300-1302, doi: 10.1038/ nsmb. 2140 .

59. Liu, G., Song, G., Zhang, D., Zhang, D., Li, Z., et al. (2014) EF-G catalyzes tRNA translocation by disrupting interactions between decoding center and codon-anticodon duplex, Nat. Struct. Mol. Biol., 21, 817-824, doi: 10.1038/nsmb.2869.

60. Abeyrathne, P. D., Koh, C. S., Grant, T., Grigorieff, N., and Korostelev, A. A. (2016) Ensemble cryo-EM uncovers inchworm-like translocation of a viral IRES through the ribosome, Elife, 5, e14874, doi: 10.7554/eLife.14874.

61. Taylor, D. J., Nilsson, J., Merrill, A. R., Andersen, G. R., Nissen, P., and Frank, J. (2007) Structures of modified eEF2 80S ribosome complexes reveal the role of GTP hydrolysis in translocation, EMBO J., 26, 2421-2431, doi: 10.1038/sj.emboj.7601677.

62. Pestka, S. (1968) Studies on the formation of transfer ribonucleic acid-ribosome complexes. 3 . The formation of peptide bonds by ribosomes in the absence of supernatant enzymes, J. Biol. Chem., 243, 2810-2820, doi: 10.1016/ S0021-9258(18)93445-9.

63. Gavrilova, L. P., Koteliansky, V. E., and Spirin, A. S. (1974) Ribosomal protein S12 and 'non-enzymatic' translocation, FEBS Lett., 45, 324-328, doi: 10.1016/0014-5793(74)80872-0.

64. Gavrilova, L. P., and Spirin, A. S. (1974) Interaction of $\mathrm{SH}$-reagents with the ribosomal $30 \mathrm{~S}$ subparticle and 'nonenzymatic' translocation, FEBS Lett., 39, 13-16, doi: 10.1016/0014-5793(74)80005-0.
65. Cukras, A. R., Southworth, D. R., Brunelle, J. L., Culver, G. M., and Green, R. (2003) Ribosomal proteins S12 and S13 function as control elements for translocation of the mRNA:tRNA complex, Mol. Cell, 12, 321-328, doi: 10.1016/s1097-2765(03)00275-2.

66. Ermolenko, D. N., Cornish, P. V., Ha, T., and Noller, H. F. (2013) Antibiotics that bind to the A site of the large ribosomal subunit can induce mRNA translocation, $R N A, \mathbf{1 9}$, 158-166, doi: 10.1261/rna.035964.112.

67. Liu, T., Kaplan, A., Alexander, L., Yan, S., Wen, J. D., et al. (2014) Direct measurement of the mechanical work during translocation by the ribosome, Elife, 3, e03406, doi: 10.7554/eLife.03406.

68. Howard, J. (2006) Protein power strokes, Curr. Biol., 16, R517-519, doi: 10.1016/j.cub.2006.06.045.

69. Hwang, W., and Karplus, M. (2019) Structural basis for power stroke vs. Brownian ratchet mechanisms of motor proteins, Proc. Natl. Acad. Sci. USA, 116, 19777-19785, doi: $10.1073 /$ pnas. 1818589116 .

70. Peske, F., Matassova, N. B., Savelsbergh, A., Rodnina, M. V., and Wintermeyer, W. (2000) Conformationally restricted elongation factor $\mathrm{G}$ retains GTPase activity but is inactive in translocation on the ribosome, $\mathrm{Mol}$. Cell, 6, 501505, doi: 10.1016/S1097-2765(00)00049-6.

71. Chen, C., Cui, X., Beausang, J. F., Zhang, H., Farrell, I., et al. (2016) Elongation factor $\mathrm{G}$ initiates translocation through a power stroke, Proc. Natl. Acad. Sci. USA, 113, 7515-7520, doi: 10.1073/pnas.1602668113.

72. Ratje, A. H., Loerke, J., Mikolajka, A., Brunner, M., Hildebrand, P. W., et al. (2010) Head swivel on the ribosome facilitates translocation by means of intra-subunit tRNA hybrid sites, Nature, 468, 713-716, doi: 10.1038/ nature 09547 .

73. Ryazanov, A. G., Natapov, P. G., Shestakova, E. A., Severin, F. F., and Spirin, A. S. (1988) Phosphorylation of the elongation factor 2 : the fifth $\mathrm{Ca}^{2+} /$ calmodulin-dependent system of protein phosphorylation, Biochimie, 70, 619626, doi: 10.1016/0300-9084(88)90245-3.

74. Ryazanov, A. G., Shestakova, E. A., and Natapov, P. G. (1988) Phosphorylation of elongation factor 2 by EF-2 kinase affects rate of translation, Nature, 334, 170-173, doi: 10.1038/334170a0.

75. Ryazanov, A. G., Rudkin, B. B., and Spirin, A. S. (1991) Regulation of protein synthesis at the elongation stage. New insights into the control of gene expression in eukaryotes, FEBS Lett., 285, 170-175, doi: 10.1016/00145793(91)80798-8.

76. Davydova, E. K., and Ovchinnikov, L. P. (1990) ADP-ribosylated elongation factor 2 (ADP-ribosyl-EF-2) is unable to promote translocation within the ribosome, FEBS Lett., 261, 350-352, doi: 10.1016/0014-5793(90)80589-B.

77. Ermolenko, D. N., and Mathews, D. H. (2021) Making ends meet: new functions of mRNA secondary structure, Wiley Interdiscip. Rev. RNA, 12, e1611, doi: 10.1002/wrna. 1611.

78. Lai, W. C., Kayedkhordeh, M., Cornell, E. V., Farah, E., Bellaousov, S., et al. (2018) mRNAs and lncRNAs intrinsically form secondary structures with short end-to-end distances, Nat. Commun., 9, 4328, doi: 10.1038/s41467-01806792-Z.

79. Ding, Y., Tang, Y., Kwok, C. K., Zhang, Y., Bevilacqua, P. C., and Assmann, S. M. (2014) In vivo genome-wide 
profiling of RNA secondary structure reveals novel regulatory features, Nature, 505, 696-700, doi: 10.1038/ nature 12756.

80. Rouskin, S., Zubradt, M., Washietl, S., Kellis, M., and Weissman, J. S. (2014) Genome-wide probing of RNA structure reveals active unfolding of mRNA structures in vivo, Nature, 505, 701-705, doi: 10.1038/nature 12894.

81. Aw, J. G., Shen, Y., Wilm, A., Sun, M., Lim, X. N., et al. (2016) In vivo mapping of eukaryotic RNA interactomes reveals principles of higher-order organization and regulation, Mol. Cell, 62, 603-617, doi: 10.1016/j.molcel.2016.04.028.

82. Lu, Z., Zhang, Q. C., Lee, B., Flynn, R. A., Smith, M. A., et al. (2016) RNA Duplex map in living cells reveals higher-order transcriptome structure, Cell, 165, 1267-1279, doi: 10.1016/j.cell.2016.04.028.

83. Sharma, E., Sterne-Weiler, T., O'Hanlon, D., and Blencowe, B. J. (2016) Global mapping of human RNA-RNA interactions, Mol. Cell, 62, 618-626, doi: 10.1016/j.molcel.2016.04.030.

84. Ziv, O., Gabryelska, M. M., Lun, A. T. L., Gebert, L. F. R., Sheu-Gruttadauria, J., et al. (2018) COMRADES determines in vivo RNA structures and interactions, Nat. Methods, 15, 785-788, doi: 10.1038/s41592-018-0121-0.

85. Roth, A., and Breaker, R. R. (2009) The structural and functional diversity of metabolite-binding riboswitches, Annu. Rev. Biochem., 78, 305-334, doi: 10.1146/annurev. biochem.78.070507.135656.

86. Giedroc, D. P., and Cornish, P. V. (2009) Frameshifting RNA pseudoknots: structure and mechanism, Virus Res., 139, 193-208, doi: 10.1016/j.virusres.2008.06.008.

87. Mauger, D. M., Siegfried, N. A., and Weeks, K. M. (2013) The genetic code as expressed through relationships between mRNA structure and protein function, FEBS Lett., 587, 1180-1188, doi: 10.1016/j.febslet. 2013.03.002.

88. Leipuviene, R., and Theil, E. C. (2007) The family of iron responsive RNA structures regulated by changes in cellular iron and oxygen, Cell. Mol. Life Sci., 64, 2945-2955, doi: 10.1007/s00018-007-7198-4.

89. Simon, A. E., and Miller, W. A. (2013) 3' cap-independent translation enhancers of plant viruses, Annu. Rev. Microbiol., 67, 21-42, doi: 10.1146/annurev-micro-092412-155609.

90. Shao, Y., Chan, C. Y., Maliyekkel, A., Lawrence, C. E., Roninson, I. B., and Ding, Y. (2007) Effect of target secondary structure on RNAi efficiency, $R N A, 13,1631-1640$, doi: $10.1261 /$ rna.546207.

91. Lu, Z. J., and Mathews, D. H. (2008) Efficient siRNA selection using hybridization thermodynamics, Nucleic Acids Res., 36, 640-647, doi: 10.1093/nar/gkm920.

92. Lu, Z. J., and Mathews, D. H. (2008) Fundamental differences in the equilibrium considerations for siRNA and antisense oligodeoxynucleotide design, Nucleic Acids Res., 36, 3738-3745, doi: 10.1093/nar/gkn266.

93. Tafer, H., Ameres, S. L., Obernosterer, G., Gebeshuber, C. A., Schroeder, R., et al. (2008) The impact of target site accessibility on the design of effective siRNAs, Nat. Biotechnol., 26, 578-583, doi: 10.1038/nbt1404.

94. Li, X., Quon, G., Lipshitz, H. D., and Morris, Q. (2010) Predicting in vivo binding sites of RNA-binding proteins using mRNA secondary structure, $R N A, \mathbf{1 6}, 1096-1107$, doi: 10.1261/rna.2017210.
95. Li, X., Kazan, H., Lipshitz, H. D., and Morris, Q. D. (2014) Finding the target sites of RNA-binding proteins, Wiley Interdiscip. Rev. RNA, 5, 111-130, doi: 10.1002/ wrna.1201.

96. Takyar, S., Hickerson, R. P., and Noller, H. F. (2005) mRNA helicase activity of the ribosome, Cell, 120, 49-58, doi: 10.1016/j.cell.2004.11.042.

97. Wen, J. D., Lancaster, L., Hodges, C., Zeri, A. C., Yoshimura, S. H., et al. (2008) Following translation by single ribosomes one codon at a time, Nature, 452, 598603, doi: 10.1038/nature06716.

98. Yusupova, G. Z., Yusupov, M. M., Cate, J. H., and Noller, H. F. (2001) The path of messenger RNA through the ribosome, Cell, 106, 233-241, doi: 10.1016/s00928674(01)00435-4.

99. Balakin, A., Skripkin, E., Shatsky, I., and Bogdanov, A. (1990) Transition of the mRNA sequence downstream from the initiation codon into a single-stranded conformation is strongly promoted by binding of the initiator tRNA, Biochim. Biophys. Acta, 1050, 119-123, doi: 10.1016/01674781(90)90151-q.

100. De Smit, M. H., and van Duin, J. (1990) Secondary structure of the ribosome binding site determines translational efficiency: a quantitative analysis, Proc. Natl. Acad. Sci. USA, 87, 7668-7672, doi: 10.1073/pnas.87.19.7668.

101. Poot, R. A., Tsareva, N. V., Boni, I. V., and van Duin, J. (1997) RNA folding kinetics regulates translation of phage MS2 maturation gene, Proc. Natl. Acad. Sci. USA, 94, 10110-10115, doi: 10.1073/pnas.94.19.10110.

102. Del Campo, C., Bartholomaus, A., Fedyunin, I., and Ignatova, Z. (2015) Secondary Structure across the bacterial transcriptome reveals versatile roles in mRNA regulation and function, PLoS Genet., 11, e1005613, doi: 10.1371/journal.pgen.1005613.

103. Khong, A., and Parker, R. (2020) The landscape of eukaryotic mRNPs, RNA, 26, 229-239, doi: 10.1261/ rna.073601.119.

104. Adivarahan, S., Livingston, N., Nicholson, B., Rahman, S., Wu, B., Rissland, O. S., and Zenklusen, D. (2018) Spatial organization of single mRNPs at different stages of the gene expression pathway, Mol. Cell, 72, 727738.e5, doi: 10.1016/j.molcel.2018.10.010.

105. Khong, A., and Parker, R. (2018) mRNP architecture in translating and stress conditions reveals an ordered pathway of mRNP compaction, J. Cell Biol., 217, 4124-4140, doi: $10.1083 /$ jcb. 201806183.

106. Maquat, L. E., Tarn, W. Y., and Isken, O. (2010) The pioneer round of translation: features and functions, Cell, 142, 368-374, doi: 10.1016/j.cell.2010.07.022.

107. Metkar, M., Ozadam, H., Lajoie, B. R., Imakaev, M., Mirny, L. A., et al. (2018) Higher-order organization principles of pre-translational mRNPs, Mol. Cell, 72, 715726.3, doi: 10.1016/j.molcel.2018.09.012.

108. Cetin, B., Song, G. J., and O'Leary, S. E. (2020) Heterogeneous dynamics of protein-RNA interactions across transcriptome-derived messenger RNA populations, J. Am. Chem. Soc., 142, 21249-21253, doi: 10.1021/ jacs.0c09841.

109. Yan, S., Wen, J. D., Bustamante, C., and Tinoco, I., Jr. (2015) Ribosome excursions during mRNA translocation mediate broad branching of frameshift pathways, Cell, 160, 870-881, doi: 10.1016/j.cell.2015.02.003. 
110. Doma, M. K., and Parker, R. (2006) Endonucleolytic cleavage of eukaryotic mRNAs with stalls in translation elongation, Nature, 440, 561-564, doi: 10.1038/nature04530.

111. Young, J. C., and Andrews, D. W. (1996) The signal recognition particle receptor alpha subunit assembles co-translationally on the endoplasmic reticulum membrane during an mRNA-encoded translation pause in vitro, EMBO J., 15, 172-181.

112. Caliskan, N., Peske, F., and Rodnina, M. V. (2015) Changed in translation: mRNA recoding by -1 programmed ribosomal frameshifting, Trends Biochem. Sci., 40, 265-274, doi: 10.1016/j.tibs.2015.03.006.

113. Tsuchihashi, Z., and Kornberg, A. (1990) Translational frameshifting generates the gamma subunit of DNA polymerase III holoenzyme, Proc. Natl. Acad. Sci. USA, 87, 2516-2520, doi: 10.1073/pnas.87.7.2516.

114. Belew, A. T., Meskauskas, A., Musalgaonkar, S., Advani, V. M., Sulima, S. O., et al. (2014) Ribosomal frameshifting in the CCR5 mRNA is regulated by miRNAs and the NMD pathway, Nature, 512, 265-269, doi: 10.1038/ nature 13429.

115. Jacks, T., Power, M. D., Masiarz, F. R., Luciw, P. A., Barr, P. J., and Varmus, H. E. (1988) Characterization of ribosomal frameshifting in HIV-1 gag-pol expression, Nature, 331, 280-283, doi: 10.1038/331280a0.

116. Kelly, J. A., Olson, A. N., Neupane, K., Munshi, S., San Emeterio, J., et al. (2020) Structural and functional conservation of the programmed -1 ribosomal frameshift signal of SARS coronavirus 2 (SARS-CoV-2), J. Biol. Chem., 295, 10741-10748, doi: 10.1074/jbc.AC120.013449.

117. Kelly, J. A., Woodside, M. T., and Dinman, J. D. (2021) Programmed -1 ribosomal frameshifting in coronaviruses: a therapeutic target, Virology, 554, 75-82, doi: 10.1016/ j.virol.2020.12.010.

118. Qu, X., Wen, J. D., Lancaster, L., Noller, H. F., Bustamante, C., and Tinoco, I., Jr. (2011) The ribosome uses two active mechanisms to unwind messenger RNA during translation, Nature, 475, 118-121, doi: 10.1038/nature10126.

119. Chen, C., Zhang, H., Broitman, S. L., Reiche, M., Farrell, I., et al. (2013) Dynamics of translation by single ribosomes through mRNA secondary structures, Nat. Struct. Mol. Biol., 20, 582-588, doi: 10.1038/nsmb. 2544.
120. Chen, J., Petrov, A., Johansson, M., Tsai, A., O’Leary, S. E., and Puglisi, J. D. (2014) Dynamic pathways of -1 translational frameshifting, Nature, 512, 328-332, doi: 10.1038/nature13428.

121. Kim, H. K., Liu, F., Fei, J., Bustamante, C., Gonzalez, R. L., Jr., and Tinoco, I., Jr. (2014) A frameshifting stimulatory stem loop destabilizes the hybrid state and impedes ribosomal translocation, Proc. Natl. Acad. Sci. USA, 111, 5538-5543, doi: 10.1073/pnas. 1403457111.

122. Tu, C., Tzeng, T. H., and Bruenn, J. A. (1992) Ribosomal movement impeded at a pseudoknot required for frameshifting, Proc. Natl. Acad. Sci. USA, 89, 8636-8640, doi: 10.1073/pnas.89.18.8636.

123. Somogyi, P., Jenner, A. J., Brierley, I., and Inglis, S. C. (1993) Ribosomal pausing during translation of an RNA pseudoknot, Mol. Cell. Biol., 13, 6931-6940, doi: 10.1128/ mcb.13.11.6931-6940.1993.

124. Lopinski, J. D., Dinman, J. D., and Bruenn, J. A. (2000) Kinetics of ribosomal pausing during programmed -1 translational frameshifting, Mol. Cell. Biol., 20, 1095-1103, doi: 10.1128/mcb.20.4.1095-1103.2000.

125. Kontos, H., Napthine, S., and Brierley, I. (2001) Ribosomal pausing at a frameshifter RNA pseudoknot is sensitive to reading phase but shows little correlation with frameshift efficiency, Mol. Cell. Biol., 21, 8657-8670, doi: 10.1128/MCB.21.24.8657-8670.2001.

126. Caliskan, N., Katunin, V. I., Belardinelli, R., Peske, F., and Rodnina, M. V. (2014) Programmed -1 frameshifting by kinetic partitioning during impeded translocation, Cell, 157, 1619-1631, doi: 10.1016/j.cell.2014.04.041.

127. Caliskan, N., Wohlgemuth, I., Korniy, N., Pearson, M., Peske, F., and Rodnina, M. V. (2017) Conditional switch between frameshifting regimes upon translation of dnaX mRNA, Mol. Cell, 66, 558-567 e554, doi: 10.1016/j.molcel.2017.04.023.

128. Bao, C., Loerch, S., Ling, C., Korostelev, A. A., Grigorieff, N., and Ermolenko, D. N. (2020) mRNA stem-loops can pause the ribosome by hindering A-site tRNA binding, Elife, 9, e55799, doi: 10.7554/eLife.55799.

129. Choi, J., O'Loughlin, S., Atkins, J. F., and Puglisi, J. D. (2020) The energy landscape of -1 ribosomal frameshifting, Sci. $A d v$., 6, eaax6969, doi: 10.1126/sciadv.aax6969. 